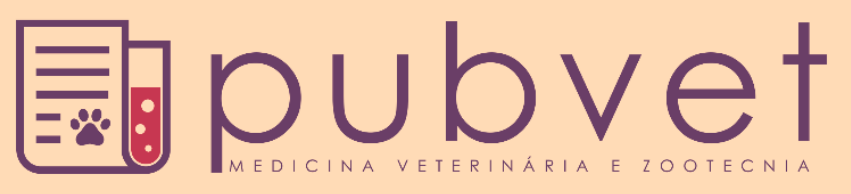

https://doi.org/10.31533/pubvet.v15n07a856.1-4

\title{
Hidrocefalia canina diagnosticada através de ultrassonografia transcraniana: Relato de caso
}

\author{
Andreza Bernardi da Silva ${ }^{1 *}$, Carina Burkert da Silva ${ }^{2}$, Thaís Cozza dos $\operatorname{Santos}^{2}{ }^{\bullet} \mathbb{0}$, \\ Eduarda Santos Bierhals ${ }^{2}$, Peter Wachholz ${ }^{30}$
}

${ }^{I}$ Graduanda na Universidade Federal de Pelotas (UFPel). Departamento de Veterinária. Pelotas-RS, Brasil.

${ }^{2}$ Médica Veterinária graduada pela UFPel. Departamento de Veterinária. Pelotas-RS, Brasil.

${ }^{3}$ Médico Veterinário graduado pela Universidade da Região da Campanha. Departamento de Veterinária. Bagé-RS, Brasil.

*Autor para correspondência, E-mail: bernardiandreza@gmail.com

\begin{abstract}
Resumo. A hidrocefalia considerada uma anomalia multifatorial, associada ao acúmulo ou passagem inadequada do líquido cefalorraquidiano, as consequências de tal enfermidade podem ser dilatação, aumento de volume e subsequente atrofia e destruição do parênquima encefálico. Seu diagnóstico definitivo se dá através de exames de imagem. Assim, tem-se como objetivo relatar o caso do diagnóstico ultrassonográfico de hidrocefalia congênita em um canino de raça Pug com 45 dias. A paciente demonstrava sinais neurológicos, como vocalização constante, head pressing e desorientação, além de atraso no desenvolvimento em relação ao restante da ninhada. Para o diagnóstico foi realizado exame ultrassonográfico da janela temporal direita, esquerda e fontanela.
\end{abstract}

Palavras-chave: Cão, diagnóstico por imagem, pug, ultrassom

\section{Canine hydrocephaly diagnosed through transcranial ultrasonography: Case report}

\begin{abstract}
Hydrocephalus is considered a multifactorial anomaly, associated with the accumulation or inadequate passage of cerebrospinal fluid, the consequences of this disease can be dilation, swelling and subsequent atrophy and destruction of the brain parenchyma. The definitive diagnosis is made through imaging exams. So, the objective is to report the case of the ultrasound diagnosis of congenital hydrocephalus in a 45-day old pug canine. The patient showed neurological signs, such as constant vocalization, head pressing and disorientation, in addition to developmental delay in relation to the rest of the litter. For diagnosis, an ultrasound examination of the right, left and fontanelle temporal windows was performed.
\end{abstract}

Keywords: Dog, imaging diagnosis, pug, ultrasound

\section{Hidrocefalia canina diagnosticada mediante ultrasonografía transcranial: Relato de caso}

Resumen. La hidrocefalia es considerada una anomalía multifactorial, asociada al acumulo o pasaje inadecuado de líquido cefalorraquídeo, las consecuencias de dicha enfermedad pueden ser dilatación, aumento de volumen y subsecuente atrofia y destrucción del parénquima encefálico. El diagnóstico definitivo se da a través de exámenes de imagen. De esta forma, se tiene como objetivo relatar el caso de diagnóstico ultrasonográfico de hidrocefalia congénita en una cachorra de la raza Pug con 45 días. La paciente demostraba signos neurológicos, como vocalización constante, head pressing y desorientación, además 
de atraso en el desarrollo en relación con el restante de los cachorros. Para el diagnóstico fue realizado examen ultrasonográfico de la ventana temporal derecha, izquierda y fontanela.

Palabras clave: Perro, diagnóstico por imagen, Pug, ecógrafo

\section{Introdução}

A hidrocefalia é uma anomalia multifatorial, associada ao acúmulo ou passagem inadequada do líquido cefalorraquidiano (LCR) desde o seu local de produção no interior do sistema ventricular até seu ponto de absorção na circulação sistêmica (Balaminut et al., 2017), tendo como consequência dilatação, aumento de volume e subsequente atrofia e destruição do parênquima encefálico (Marcondes et al., 1992). Ainda, deve-se ressaltar que esta enfermidade pode ser classificada como adquirida ou congênita, sendo a segunda de maior ocorrência e frequentemente relatada em caninos de raças pequenas e braquiocefálicos (Trindade et al., 2019).

Quanto aos sinais clínicos dessa patologia, são de demasia variedade e podem alternar conforme o local de compressão e o grau de aumento de pressão intracraniana. No entanto, as manifestações clínicas neurológicas são regularmente observadas nos caninos (Silva et al., 2016). O tratamento para tal anomalia depende da causa primária, podendo este ser clínico ou cirúrgico, considerando o estado físico e clínico do paciente. O prognóstico da hidrocefalia em cães é de reservado a ruim, baseado nos sinais clínicos apresentados pelo animal e a causa da hidrocefalia (Kent et al., 2016).

O diagnóstico da hidrocefalia é definido por uma detalhada anamnese, exame clínico e auxiliado por exames complementares, como a radiografia (RX), ultrassonografia (US), tomografia computadorizada (TC) e a ressonância magnética (RM) (Ludwig et al., 2015). Como método de primeira escolha na rotina veterinária, é preconizado o uso da radiografia, embora não contribua consideravelmente para diagnosticar definitivamente tal enfermidade (Carvalho et al., 2007; Carvalho \& Chammas, 2008). Em caso da fontanela bregmática persistente ou em determinados casos utilização do osso temporal como janela acústica, a ultrassonografia se torna o meio mais viável para o diagnóstico de hidrocefalia (Cintra et al., 2014).

O estudo foi realizado para relatar o caso do diagnóstico ultrassonográfico de hidrocefalia congênita em um canino de raça Pug com 45 dias.

\section{Material e métodos}

Foi atendido em uma clínica particular, uma fêmea canina da raça Pug com 45 dias de vida. Durante a anamnese, o tutor relatou que a paciente demonstrava sinais neurológicos, como vocalização constante, head pressing e desorientação, além de atraso no desenvolvimento em relação ao restante da ninhada.

No decorrer da realização do exame clínico-físico foi observado um aumento de volume assimétrico na região da fontanela no paciente em questão, popularmente conhecida como moleira. Os demais parâmetros fisiológicos apresentavam-se sem alteração dignas de nota. Tendo em vista os sinais apresentados pelo filhote, foi suspeitado de hidrocefalia congênita, dessa forma, foi prosseguindo através da solicitado de exame ultrassonográfico transcraniano.

\section{Resultados e discussão}

O exame ultrassonográfico foi realizado por meio da utilização de um aparelho $\mathrm{GE}^{\circledR}$, modelo Logiq E com transdutor linear multifrequencial de 8 à $13 \mathrm{MHz}$. A paciente foi contida em estação, assim, de maneira inicial, foi posicionado o transdutor de forma perpendicular ao osso temporal direito, realizando movimento contínuo rotacionado de $45^{\circ}$, seguido de movimento ventrodorsal, ou movimento de leque, para uma análise completa das estruturas cerebrais. Subsequentemente, foi realizado o mesmo procedimento na janela temporal esquerda e na fontanela.

No decurso da varredura encefálica foi observado acentuada dilatação do ventrículo lateral direito preenchido por conteúdo anecóico e homogêneo (Figura 1), como consequência, diminuição do 
ventrículo lateral esquerdo (Figura 2), bem como assimetria dos ventrículos cerebrais junto de preservação de definição anatômica das estruturas encefálicas (igura 3).

Com base na anamnese, exame clínico e resultado do exame de imagem, o diagnóstico definitivo foi de hidrocefalia congênita. A partir do diagnóstico, o paciente apresentou importante piora em curto espaço de tempo, optando-se assim para realização de eutanásia do mesmo.

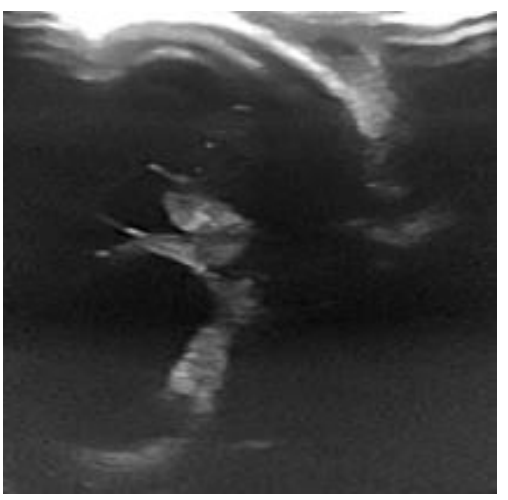

Figura 1. Janela acústica temporal esquerda evidenciando aumento do ventrículo lateral direito.

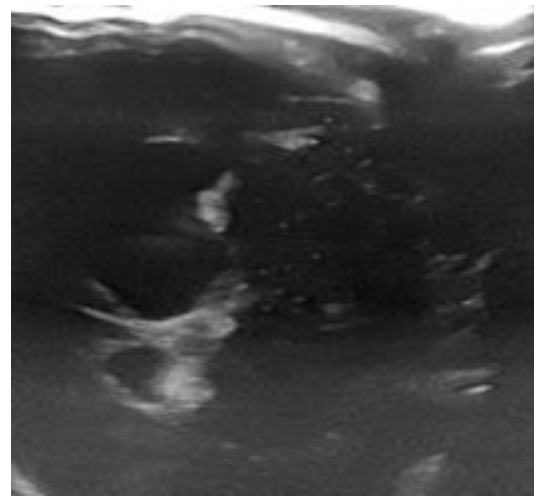

Figura 2. Janela acústica temporal direita demonstrando aumento do ventrículo lateral direito.

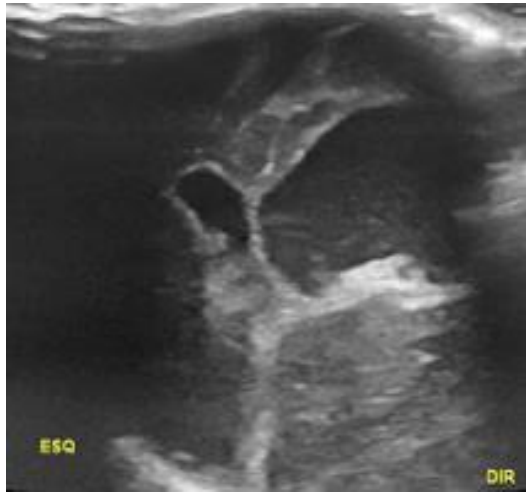

Figura 3. Imagem realizada através da fontanela, constatando assimetria dos ventrículos cerebrais.

Anomalias congênitas são apontadas em estudos de prevalência de patologias diagnosticadas na rotina clínica veterinária em cães constituindo cerca de $6 \%$ do total das doenças. Dentro dessa porcentagem, a hidrocefalia representa metade dos diagnósticos, representando uma das enfermidades mais comuns em caninos (Balaminut et al., 2017). A hidrocefalia é a distensão do sistema ventricular cerebral relativa a passagem indevida do LCR, frequentemente relatada em cães de raças pequenas e braquicefálicas, apresentando sinais clínicos até 2 anos de idade, em sua maioria sendo manifestações neurológicas, tendo como maior causa ser uma enfermidade congênita (Trindade et al., 2019), fatos estes compatíveis com o presente relato.

Apesar de alguns enfermos possam continuar estáveis por determinado tempo, os sinais clínicos na maioria dos casos têm evolução rápida, sendo inevitável optar-se pela eutanásia (Marcondes et al., 1992; Quessada et al., 2014). No caso em questão, a eutanásia foi procedida devido ao avanço dos sinais neurológicos e ao comprometimento do bem-estar animal.

$\mathrm{O}$ diagnóstico de hidrocefalia em animais in vivo é realizado preferencialmente por exames de imagem, como o RX, US, TC e RM (Belotta et al., 2013). Apesar de classificados como padrões ouro para o diagnóstico de tal enfermidade, a TC e RM, não são considerados ordinários e cotidianos nos hospitais e clínicas veterinárias brasileiras. Assim, tornando o RX e US métodos mais acessíveis (Ludwig et al., 2015). Quanto a radiografia em casos de deformação craniana, ainda é o método mais utilizado como escolha para diagnóstico de hidrocefalia pelo médico veterinário, mesmo na eventualidade de que a radiografia convencional não colabore expressivamente para tal diagnóstico, em virtude da sua insensibilidade aos tecidos moles (Cintra et al., 2014).

A ultrassonografia trans craniana (USTC), diferentemente da medicina veterinária, na neonatologia humana apresenta-se como a categoria primeiramente adotada em casos de suspeita de hidrocefalia, tanto em neonatos quanto em crianças (Carvalho et al., 2007). Fato este sustentado devido a USTC ser um recurso o qual não utiliza radiação ionizante, podendo ser utilizada de maneira periódica sem originar danos à saúde do paciente, além de apresentar baixo custo e amplo valor diagnóstico, especialmente devido sua rapidez e não ser um método invasivo, não necessitando de anestesia para sua realização (Cintra et al., 2014). Tais aspectos levados na escolha da utilização da USTC no paciente do caso.

\section{Conclusão}

Mesmo com os avanços científicos apresentados no decorrer dos anos, há um hiato na literatura relativo à aplicação da ultrassonografia trans craniana utilizada como meio de diagnóstico em cães para 
tal enfermidade na rotina médico-hospitalar, bem como uma escassez dos relatos. Assim, no presente trabalho, a ultrassonografia trans craniana manifestou-se um instrumento útil para o diagnóstico de hidrocefalia, corroborando para diminuição da carência de casos apresentados na literatura.

\section{Referências}

Balaminut, L. F., Pires, A. C. M., \& Troncarelli, M. Z. (2017). Canine congenital hydrocephalus followed by an iatrogenic hiperadrenocorticism status: review and case report. Veterinaria $e$ Zootecnia, 24(4), 639-650.

Belotta, A. F., Machado, V. M. de V., \& Vulcano, L. C. (2013). Diagnóstico da hidrocefalia em animais através da ultrassonografia, tomografia computadorizada e ressonância magnética. Veterinária $e$ Zootecnia, 20(1), 33-41.

Carvalho, C. F., Andrade Neto, J. P., Jimenez, C. D., Diniz, S. A., Cerri, G. G., \& Chammas, M. C. (2007). Ultra-sonografia transcraniana em cães com distúrbios neurológicos de origem central. Arquivo Brasileiro de Medicina Veterinária e Zootecnia, 59(6), 1412-1416.

Carvalho, C. F., \& Chammas, M. C. (2008). Uso do ultrassom duplex Doppler no diagnóstico de shunt portossistêmico em gatos. Arquivo Brasileiro de Medecina Veterinária e Zootecnia, 60(1), 109-112.

Cintra, T. C. F., Carvalho, C. F., Canola, J. C., \& Nepomuceno, A. C. (2014). Ultrassonografia transcraniana em cães hígidos: padronização da técnica e descrição anatômica. Arquivo Brasileiro de Medicina Veterinária e Zootecnia, 66(1), 61-68.

Kent, M., Glass, E. N., Haley, A. C., Shaikh, L. S., Sequel, M., Blas-Machado, U., Bishop, T. M., Holmes, S. P., \& Platt, S. R. (2016). Hydrocephalus secondary to obstruction of the lateral apertures in two dogs. Australian Veterinary Journal, 94(11), 415-422.

Ludwig, M. P., Teichmann, C. E., \& Serafini, G. M. C. (2015). Ultrassonografia transcraniana em um cão com hidrocefalia. Salão Do Conhecimento, 1, 1-4.

Marcondes, M., Dagli, M. L. Z., Iwasaki, M., \& Santis Prada, I. L. (1992). Hidrocefalia congênita em cão: revisão de literatura e relato de caso clínico. Brazilian Journal of Veterinary Research and Animal Science, 29(1), 105-112.

Quessada, A. M., Zamarian, T., Paula, D., Ribeiro, R. C., \& Dias, S. H. (2014). Hidrocefalia em cão: Relato de caso. Enciclopédia Biosfera, 10(19), 1-9.

Silva, S., Castro, M., Wilson, T., Medeiros-Ronchi, A., \& Costa, F. (2016). Hidrocefalia congênita em cão - relato de dois casos. Enciclopédia Biosfera, 13(24), 580-586.

Trindade, A. B., Sá, T. C., Pessoa, L. F., Trindade, A. B., Fernandes, E. P. A., \& Paula, D. S. (2019). Hidrocefalia canina-relato de caso. Arquivos de Ciências Veterinárias e Zoologia Da UNIPAR, 22(2), 65-68.

Histórico do artigo:

Recebido: 4 de fevereiro de 2021

Aprovado: 19 de março de 2021.
Licenciamento: Este artigo é publicado na modalidade Acesso Aberto sob a licença Creative Commons Atribuição 4.0 (CC-BY 4.0), a qual permite uso irrestrito, distribuição, reprodução em qualquer meio, desde que o autor e a fonte sejam devidamente creditados. 